Cardiol Young 2000; 10: 75-79

(C) Greenwich Medical Media Ltd ISSN 1047-95 11

\title{
1. Newsletter from the Association for European Paediatric Cardiology
}

\section{From old to new}

\author{
Dr. O. Daniëls (Secretary-General, Association for European Paediatric Cardiology)
}

$\mathrm{T}$ he past year was an exiting one. It was the last year of this century and millennium. It was the first time we were forced to cancel a General Annual Meeting because of war. Due to the enormous efforts of Vladimir Pilossoff, along with his colleagues and congress organisers, we were subsequently able to reorganise and hold the Annual General Meeting, from September $29^{\text {th }}$ to October $2^{\text {nd }}$, in Sofia, Bulgaria. The meeting was characterised by its good scientific content, as well as its pleasant contacts, and was made perfect by the smooth organisation. It was important that we were able to hold this meeting because we had to decide on some vital issues. Many things concerning the Association had already been planned, but awaited ratification.

First, there was a planned change in the Constitution so as to make membership more open for our colleagues living outside of Europe. The old definition of "Corresponding membership" was deemed to be too restrictive, not only in terms of providing satisfactory exchange of thoughts and expertise, but also in respect of collegial contacts. At the Business meeting of October the $2^{\text {nd }}$, the General assembly voted in favour of the proposed change, namely to "Medical and other scientific graduates who are committed to the practice and advancement of Paediatric Cardiology and closely related fields living outside Europe may be elected to Corresponding Membership".

A second crucial ratification was the acceptance of the European Paediatric Cardiology Code, with which readers of the Journal are already familiar. The short list of the code was published in the final issue of 1999. The long list is published as a supplement together with this issue. The combined lists permit all diagnostic, as well as therapeutic, items in the field of paediatric cardiology and cardiac surgery to be coded in precise and unambiguous fashion. It is

Correspondence to: Otto Daniels, Secretary-General, Association for European Paedıatric Cardiology, University Hospital Nijmegen, Kinderhartcentrum, Postbus 9101, 6500 HB Nijmegen, The Netherlands the Association for European Paediatric Cardiology which holds the copyright for this system, and which accepts the responsibility for maintaining its future integrity. The lists are also available from our Internet site at www.aepc.org. Everybody in the field of paediatric cardiology is now free to use these codes for their own purpose.

The third crucial decision was the acceptance of the new requirements for training. These are also published in this issue of the Journal, immediately following this letter. As an Association, we first produced written agreements on requirements for training in 1990. It was clear from the beginning that these requirements would require updating. So, in 1996, the Association introduced the mechanisms for revising the requirements, using the assembly of National Delegates for this purpose. From this assembly, a sub-committee was formed under the presidency of Carlo Kallfelz, consisting of Albrecht Beitzke, Desmond Duff, Joes Ramsoe Jacobsen, Jaap Ottenkamp, Erki Pesonen and Fernando Picchio, who were set the task to provide the necessary update. Their proposals were discussed extensively in the subsequent meetings of the National Delegates. The final proposal was then put before the members present in Sofia for ratification.

New discussions are now underway concerning the criterions to be met by those institutes providing training, and by those offering themselves as trainers in the field of paediatric cardiology, as well as recommendations for continuing medical education. The initial proposals have already been distributed by the new secretary of the Advisory Professional Committee and National Delegates, Béat Friedli, to the National Delegates, and are available for scrutiny on our web page. So, everybody can take notice of them. They will be discussed through the coming year, and submitted for ratification at Strasbourg during our next Annual General Meeting for 2000.

In 1999, we have also looked again at the activities in our field, re-exploring the questions initially 
posed in 1991. Needless to say, the results are crucially important for our progress. They will help define in a better way the guidelines for training, those institutes considered appropriate for training, and how best to provide optimal care for patients.

The year 2000, and those which will immediately follow, are likely to prove crucial in constructing and defending our profession, as they will for all medical specialties. It is no secret that, in these years, we hope to establish paediatric cardiology as a fully recognised specialty in its own right. As we start this new millennium, the Association has already constructed the solid base for this much needed progress.

\section{Guidelines for training in paediatric cardiology}

\section{Duration of training}

In order to be recognized as a general paediatric cardiologist, it is necessary for the trainee to complete a full time period of training of seven years. For 3 to 4 years of this training, the trainee should be exposed to all aspects of cardiovascular medicine as the patients studied grow from fetal life to become adolescents and adults. During this period of the 3 to 4 years of full-time training in paediatric cardiology, up to six months may be spent in either adult cardiology, cardiac surgery, cardiac pathology, respiratory physiology, or anaesthesiology. One year of the total period of training can be devoted to research. In order to enter specialist training in paediatric cardiology, the trainee should have completed either of the following periods of postgraduate training:

- 3 to 4 years in general paediatrics, including at least 6 months of neonatology and neonatal intensive care

- 2 to 3 years in general paediatrics, including at least 6 months neonatology, and a further 1 to 2 years in adult cardiology.

It should be recognised that the schedule of training detailed above is the minimum required to become a paediatric cardiologist. For some specific subspecialties (see the addendum), additional training is mandatory. The trainee must keep a logbook in which it states that the trainee has satisfactorily completed these requirements.

\section{Basic requirements and objectives of training}

Programmes designed to train paediatric cardiologists need to provide an intellectual environment for acquiring the knowledge, skill and clinical judgement essential for this specialty. Fundamental goals of this training are the provision of the best possible care for each patient, and a compassionate attitude to the patients and their parents or guardians.

The programmes should have an appropriate balance between clinical service and academic endeavours, and should encourage the trainees to become dedicated to continuous education and teaching. Critical thinking, and deeper insight into the practical and theoretical problems of the speciality, can be improved by active participation in research. The trainees, therefore, are expected to undertake clinical or basic research, and to publish their findings in peer reviewed journals.

\section{Contents and targets of the specialist training}

Basic knowledge and skills

Embryology, teratology, normal and morbid anatomy of the cardiovascular system in the growing individual

A basic understanding of these morphologic aspects should be achieved in the first year, and may be obtained by attendance at an approved foundation course, which should include teaching of the current nomenclature. Thereafter, the trainee should take the opportunity regularly to review specimens under expert guidance.

\section{Normal and pathological physiology of the cardiovascular system}

Whilst the principles of cardiovascular physiology may be obtained by reading, this is no substitute for personally performing studies. The knowledge thus acquired should then be supplemented by observing, assisting in, and evaluating haemodynamic assessment by cardiac catheterization and by cross-sectional and Doppler echocardiography. Further knowledge and experience in the haemodynamic monitoring and pathophysiology of the cardiovascular system may be obtained by spending time in the intensive care unit.

\section{Epidemiology, human genetics, medical statistics}

These three sub-specialities are important for the theory, practice, and teaching in academic medicine. The trainee, preferably in the first year of training, should become well acquainted with these aspects by reading around the subjects, and by attending foundation courses. 
Cardiovascular pharmacology

Knowledge of the effects, and the side-effects, of cardiovascular drugs, and their interaction with other pharmacological substances in different diseases and at different ages, should be acquired early in the training.

Aetiology, symptomatology and clinical diagnosis and differential diagnosis of congenital and acquired disease of the cardiovascular system

The prerequisite of a sound practice of paediatric cardiology is the precise knowledge of the cardiovascular symptoms and signs and their interpretation, as well as their general and specific effects on other systems. The necessary experience must be gained by extensive participation in the daily clinical work of the department, studying both in-patients and out-patients. At the start of the training, this should be under the supervision and guidance of an experienced paediatric cardiologist, from whom the trainee can learn the basic arts of history taking and physical examination.

\section{Specific technical skills in paediatric cardiology \\ Electrocardiography}

Interpretation and formal reporting of the electrocardiogram should start early in the first year, and should be audited by one of the trainers. By the end of the training, the trainee should have reported on about 1000 electrocardiographic recordings.

The trainee should obtain experience in the evaluation of arrhythmias. 24-hour tapes should be interpreted, and reported, as soon as basic knowledge has been gained in cardiac arrhythmias. After 3 years of training, the trainee should have documented the personal reporting of 10024 -hour tapes.

\section{Cardiac ultrasound}

Echocardiography, and Doppler echocardiography, are an integral part of paediatric cardiology. They are the most important non-invasive techniques for diagnosis, clinical management and follow-up of heart disease in the young. The trainee, therefore, should gain experience in these techniques in the first year. It is recommended that the trainee should attend a foundation course on echocardiography in the first year of the training.

After this, an integral program of performing and reporting on echocardiograms should be developed by the trainee. The reporting of scans by the trainee should be audited and certified by one of the trainers. At the end of the training period, at least 1000 echocardiographic examinations, including M-mode, cross-sectional, Doppler, colour Doppler, transesophageal, fetal interrogations, 3-dimensional reconstructions, and so on, should have been performed and interpreted personally, and should have been certified by the trainer.

The early recognition and management of congenital cardiac malformations, or disturbances of rhythm in pregnancies at risk, is dependent on fetal echocardiography performed by an expert examiner. The trainee with expertise in routine echocardiography, usually after the second year of training, should therefore be exposed to as much fetal echocardiography as is possible. The number of fetal echocardiograms performed and interpreted should be kept in a logbook. The scans should include fetuses with normal and abnormal hearts, and these should all be accurately documented. The trainee should also participate in counselling sessions for the parents with of fetuses shown to have abnormal hearts. Fetal echocardiography may be performed in cooperation with obstetricians, or in the department of paediatric cardiology.

Stress echocardiography is an additional ultrasonic method which should be considered as optional, and which the candidate should be familiar with, but this technique may be learnt on secondment to a unit specialising in adult cardiology.

\section{Exercise testing}

School children and adolescents with various types of congenital and acquired heart diseases, but mainly after surgery for correction or palliation of congenital heart defects, may require testing by bicycle or treadmill ergometry for the evaluation of their functional status and the detection of exercise-induced arrhythmias. A minimum of 40 exercise testing examinations should be performed. Exposure to these should commence in the first year of the period of training. The method may be learnt quickly on secondment to a unit specialising in adult cardiology or respirology.

\section{Cardiovascular radiology and nuclear cardiology}

Chest x-ray

At the outset of training, the trainee should interpret chest radiographs of the patients being managed. The reading and reporting of the films should be supervised by a paediatric cardiologist, or by a cardiovascular radiologist. By the end of the period of training, at least 200 chest radiographs should have been reported by the trainee and certified by the trainer. 
Cardiovascular Computerised Tomographic Scanning and Resonance Imaging

These non-invasive techniques play a growing role in the pre- and postoperative evaluation of patients with congenital heart disease, serving to supplement the various echocardiography techniques. The trainee should become familiar with the methodologies, the diagnostic potential, and the limitations of these additional techniques. This goal can be reached by participating for a period of two months in the daily work of a department specialising in radiology and nuclear medicine and regularly investigating patients with cardiac disease. Another approach would be to attend a foundation course in these techniques, and then to be involved regularly in the imaging of patients known to the trainee. Participation in 20 sessions is required.

\section{Other techniques of nuclear cardiology}

The trainee should acquire knowledge concerning the indications, the diagnostic value and limitations, and the practical techniques of procedures such as myocardial imaging by means of SPECT and positron emission tomography, and studies of ventricular function, as well as investigations of lung perfusion and ventilation.

\section{Cardiac catheterisation and angiocardiography}

Haemodynamic investigations

After gaining experience in the non-invasive techniques, the trainee should learn the technique of cardiac catheterization. The trainee should initially work as an assistant to an experienced investigator, thus coming to learn and understand the basics of the technique before being allowed to perform investigations, first under supervision, and then independently. Besides learning the technical details, it is essential that the trainee becomes conversant personally with calculation of hemodynamic shunts and resistances, and is able to present the data during staff meetings. A minimum of 100 diagnostic procedures should be performed independently, and audited and certified by the trainer.

\section{Angiocardiography}

This method is an integral part of diagnostic cardiac catheterisation, and should be taught in conjunction with the haemodynamic study. The trainee should learn the method of angiocardiography, its benefits and limitations, as well as its associated risks. The trainee should learn the various angled views required to profile the different cardiac structures.
Of necessity, the techniques of cardiac catheterisation and angiocardiography will be encountered throughout the period of training. The trainee should have performed these independently in at least 100 patients, and these investigations should be certified by the trainer.

\section{Interventional catheterisation}

When competence in diagnostic cardiac catheterisation and angiocardiography has been achieved, the trainee should be exposed to the common interventional procedures, such as balloon atrial septostomy, balloon dilation of valves or arteries, closure of intracardiac and extracardiac defects and vessels, and implantation of stents. At the completion of the training, a minimum of 25 varied interventional procedures should have been performed and reported on under supervision, and audited and certified by the trainer.

\section{Diagnosis and treatment of cardiac rbythm disorders and electrocardiology}

Diagnosis of arrhythmias

In addition to obtaining the basic understanding of electrocardiography discussed above, the trainee should become familiar with the electrophysiologic mechanisms and underlying aetiologies of disorders of cardiac thythm. This can be supplemented by attending a foundation course on electrophysiology, or by taking part in the non-invasive and invasive electrophysiologic evaluation of patients with arrhythmias.

\section{Drug treatment of disorders of rhythm}

The number of drugs used for the treatment of arrhythmias is vast, and the effects and side-effects are numerous. The trainee should, therefore, acquire detailed knowledge in this field. It is advisable to attend a foundation course. It is also important to be closely involved in the clinical management of the arrhythmias. The progress of the trainee should be certified by the trainer.

\section{Cardioversion of tachyarrhythmias}

Termination of atrial and ventricular tachycardias by direct current shock, especially in emergency situations, is an important therapeutic technique, and should be learned within the first few months of training. At the outset of the training, the trainee should be taught under supervision. Subsequently, the trainee should perform independently cardioversion of 
atrial tachycardias, either by transesophageal or transvenous overdrive pacing, or by programmed stimulation. In postoperative patients, this may be accomplished by using external epicardial electrodes. A minimum of 20 electrocardioversions should have been certified by the end of the period of training.

\section{Cardiac Pacing}

From the beginning of the period of training, the trainee should become familiar with the indications for, and the different modes of, cardiac pacing, as well as the insertion of endocardial pacing electrodes for emergencies.

\section{Noninvasive therapy of cardiovascular diseases}

Besides drug treatment of disorders of rhythm, the trainee should acquire sound theoretical knowledge and practical experience of the management of heart failure and hypertension using drugs in infancy and childhood. The trainee should have been responsible for the investigation and management of these patients.

\section{Care of Patients}

\section{Inpatients}

During the entire period of training, the trainee should have been involved in the management of, and have been responsible for, at least 300 patients. These should include patients in the early postoperative period, and those admitted for long-term follow-up.

\section{Outpatients}

It is recommended that the trainee takes part in outpatient care either regularly over the whole training or for a certain fixed period of time, and that the trainee has seen at least 1150 patients in the outpatient clinics.

\section{Intensive care \\ In addition to the obligatory 6 months of neonatal intensive care, it is mandatory that at least 6 months are also spent in an intensive care unit, where the trainee should have taken personal responsibility for the management of pre-, postoperative, or postinter- ventional patients with cardiac disease. This will ensure adequate knowledge and practical skills for the management of this often difficult group of patients.}

\section{On-call commitment}

From the second year of training onwards, the trainee should be able to participate in the on-call schedules.
To ensure adequate experience, especially in the management of emergency admissions, the trainee should be regularly on call no more than twice a week throughout the total period of training.

\section{Certification \\ Certification of activities}

The trainee should keep an accurate personal logbook of all activities undertaken over the entire period of training. It is the responsibility of the trainer to audit and certify special activities as identified in the above paragraphs.

\section{Annual evaluation}

An annual evaluation of progress of the trainee should be performed by the head of the department providing the programme of training.

\section{Board certification}

The Association for European Cardiology will issue a certificate stating that a trainee has fulfilled the requirements for training subsequent to evaluation of the programme of training, scrutiny of the logbook, and the statement of the trainer(s) that the candidate had been trained according to the guidelines set out above.

In the future, a formal final examination, containing both written and oral parts, will be deemed mandatory to assess the clinical skills and the theoretical knowledge.

\section{Addendum}

There are some areas of Paediatric Cardiology requiring experience in additional sub-specialties. This additional experience can be acquired in larger centres. The areas include:

- Therapeutic catheter interventions.

- Cardiac electrophysiology, including mapping and ablative procedures, as well as implantation of multi-programmable pacemakers.

- Fetal cardiology.

- Specialisation in echo-Doppler cardiology, for example transoesophageal, and 3-dimensional echocardiography.

Centres performing this type of work should be committed to providing extensive postgraduate training, and to offer time-limited training positions and regular courses. 